\title{
Tijolo de solo-cimento: incorporação de resíduos e viabilidade na construção civil no
}

\section{Brasil}

\author{
Soil-cement brick: incorporation of waste and feasibility in civil construction in Brazil \\ Ladrillo suelo-cemento: incorporación de residuos y viabilidad en la construcción civil en Brasil
}

Recebido: 11/01/2022 | Revisado: 15/01/2022 | Aceito: 20/01/2022 | Publicado: 25/01/2022

Bruno Sanches da Silva

ORCID: https://orcid.org/0000-0002-2297-3810 Universidade Estadual do Norte Fluminense Darcy Ribeiro, Brasil E-mail: bbrunosanchesl@gmail.com

Natália Tavares Gomes

ORCID: https://orcid.org/0000-0003-2183-8198 Universidade Estadual do Norte Fluminense Darcy Ribeiro, Brasil E-mail: natalia.t.gomes@hotmail.com

Alexandre Vianna Bahiense

ORCID: https://orcid.org/0000-0002-4678-1709 Instituto Federal do Espírito Santo, Brasil E-mail: abahiense@ifes.edu.br

Rafael Picanço de Oliveira

ORCID: https://orcid.org/0000-0003-2273-9976 Instituto Federal Fluminense, Brasil E-mail: oliveirarp@live.com

Jonas Alexandre

ORCID: https://orcid.org/0000-0002-2977-5585 Universidade Estadual do Norte Fluminense Darcy Ribeiro, Brasil E-mail: jonasuenf@gmail.com

\begin{abstract}
Resumo
A busca por materiais de menor impacto ambiental é pauta fundamental para uma constante evolução da construção civil e sociedade, tornando-se cada vez mais recorrente. Esses materiais precisam apresentar uma diminuição da emissão de poluentes na atmosfera, cumprir os requisitos técnicos que as normas estabelecem em função da finalidade de cada composição, possibilitar redução ou reaproveitamento de resíduos, e uma boa relação custo-benefício. Desse modo, o presente artigo objetiva analisar a viabilidade econômica e ambiental do tijolo de solo-cimento na construção civil no Brasil e identificar os principais resultados da incorporação de resíduos em sua fabricação. Trata-se de uma revisão bibliográfica integrativa da literatura, que foi realizada entre agosto de 2021 a janeiro de 2022 . O tijolo de solo-cimento apresenta uma economia de $41 \%$ comparado ao tijolo cerâmico, podendo ser articulado com as habitações de interesse social; além disso, gera menos impacto ambiental, seja pela diminuição dos gases poluentes - redução de $29,5 \%$ de $\mathrm{CO}_{2}$, ou pelo reaproveitamento de resíduos. É possível encontrar na literatura a incorporação de diversos resíduos com resultados diferentes. Portanto, o potencial do tijolo de solo-cimento fica evidente pelo reaproveitamento de resíduos, auxiliando no descarte e reaproveitamento; por ter um menor custo financeiro da obra e menor emissão de $\mathrm{CO}_{2}$; além de ter potencial para ser utilizado em programas habitacionais com intuito de diminuir o déficit habitacional no Brasil. No entanto, são necessárias mais pesquisas com esse método construtivo, com a finalidade de adquirir uma maior confiabilidade desse material.
\end{abstract}

Palavras-chave: Tijolo de solo-cimento; Construção civil; Resíduos da construção civil.

\begin{abstract}
The search for materials with lower environmental impact is a fundamental agenda for a constant evolution of civil construction and society, becoming increasingly recurrent. These materials have to present a reduction in the atmosphere pollutants emission, fulfill the technical requirements established by the standards according to the purpose of each composition, enable to reduce or reuse of waste, and a good cost-benefit ratio. Thus, this article aims to analyze the economic and environmental viability of the soil-cement brick in civil construction in Brazil, as well as to identify the main results of the incorporation of waste in its manufacture. This is an integrative bibliographic review of the literature, which was carried out between August 2021 and January 2022. The soil-cement brick has an economy of $41 \%$ when compared to ceramic brick, and can be articulated with the social interest housing; in addition, it generates less environmental impact, either by reducing polluting gases - a $29.5 \%$ reduction in $\mathrm{CO}_{2}$, or by reusing waste. It is possible to find in the literature the incorporation of several residues with different results. Therefore, the potential of the soilcement brick is evident by the reuse of waste, helping in the disposal and reuse; for having a lower financial cost in the
\end{abstract}


construction and lower $\mathrm{CO}_{2}$ emission; in addition to having the potential to be used in housing programs in order to reduce the housing deficit in Brazil. However, more research is needed with this constructive method, in order to acquire greater reliability of this material.

Keywords: Soil-cement brick; Civil construction; Civil construction waste.

\section{Resumen}

La búsqueda de materiales con menor impacto ambiental es una agenda fundamental para una constante evolución de la construcción civil y la sociedad, cada vez más recurrente. Estos materiales deben presentar una reducción en la emisión de contaminantes a la atmósfera, cumplir con los requisitos técnicos establecidos por las normas según el propósito de cada composición, permitir la reducción o reutilización de residuos y una buena relación costo-beneficio. Así, este artículo tiene como objetivo analizar la viabilidad económica y ambiental del ladrillo de suelo-cemento en la construcción civil en Brasil e identificar los principales resultados de la incorporación de residuos en su fabricación. Esta es una revisión bibliográfica integradora de la literatura, que se realizó entre agosto de 2021 y enero de 2022 . El ladrillo de suelo-cemento tiene un ahorro del $41 \%$ frente al ladrillo cerámico, y puede articularse con vivienda de interés social; además, genera un menor impacto ambiental, ya sea por la reducción de gases contaminantes -una reducción del $29,5 \%$ de $\mathrm{CO}_{2}$, o por la reutilización de residuos. Es posible encontrar en la literatura la incorporación de varios residuos con diferentes resultados. Por lo tanto, se evidencia el potencial del ladrillo de suelo-cemento por la reutilización de residuos, auxiliando en la disposición y reutilización; por tener un menor coste económico de la obra y menor emisión de $\mathrm{CO}_{2}$; además de tener el potencial de ser utilizado en programas de vivienda para reducir el déficit habitacional en Brasil. Sin embargo, se necesita más investigación con este método constructivo, con el fin de adquirir una mayor fiabilidad de este material.

Palabras clave: Ladrillo de suelo-cemento; Construcción civil; Residuos de la construcción.

\section{Introdução}

A busca por materiais de menor impacto ambiental, que apresentam uma diminuição da emissão de poluentes na atmosfera, que possibilitam uma redução ou reaproveitamento de resíduos, com uma boa relação custo-benefício e que possam ser utilizados na construção civil de forma a cumprir os requisitos técnicos que as normas estabelecem, em função da finalidade de cada composição, são pautas fundamentais para uma constante evolução da construção civil e sociedade, tendo se tornado cada vez mais recorrente.

Em meio a esse cenário de grande preocupação ambiental, a estabilização de solo com destino à utilização na construção civil, como na produção de tijolos de solo-cimento, surge como uma alternativa viável, factível e de menor impacto ambiental face aos métodos construtivos já consolidados. Para ter oportunidade de tal ascensão no mercado, é necessário que o tijolo de solo-cimento conquiste mais confiabilidade e credibilidade, que até então não possui de forma expressiva.

Na construção civil, além dos problemas ambientais, há o problema do déficit habitacional no Brasil; um estudo publicado pela Fundação João Pinheiro (FJP), relata que o déficit habitacional estimado no território Brasileiro para o ano de 2019 foi de 5,876 milhões de domicílios; o que representa, em percentual relativo, 8\% do total de domicílios particulares permanentes e improvisados do país (FJP, 2021).

Existem programas destinados à habitação social no país, como Minha Casa Minha Vida (MCMV), Pró-Moradia e Agência Paulista de Habitação Social (Casa Paulista), além de parcerias privadas e públicas; no entanto, ao olhar para os levantamentos estatísticos da FJP (2021) e suas perspectivas, percebe-se que ainda não são suficientes, uma vez que houve um aumento no déficit habitacional de 2016 para 2019 (4\%) e é esperado um aumento para 2021 e próximos anos.

Portanto, visando uma constante evolução da construção civil e sociedade, considerando o potencial e as consequências do uso do tijolo de solo-cimento, é primordial o desenvolvimento de pesquisas com esse método construtivo, com a finalidade de adquirir uma maior confiabilidade desse material. Nesse sentido, o presente artigo objetiva analisar a viabilidade econômica e ambiental do tijolo de solo-cimento na construção civil no Brasil, assim como identificar os principais resultados da incorporação de resíduos em sua fabricação. 


\section{Metodologia}

O presente estudo configura-se como uma revisão bibliográfica integrativa da literatura, realizado entre agosto de 2021 a janeiro de 2022. A revisão integrativa da literatura possibilita a síntese do conhecimento e a análise crítica dos resultados de estudos relevantes e indícios sobre algum conteúdo definido (Whittemore \& Knafl, 2005).

Para o desenvolvimento da pesquisa, foi obedecido as seguintes etapas: definição do problema de pesquisa; busca no Portal periódicos CAPES, por meio da comunidade acadêmica federada (cafe); seleção dos artigos; resultados e discussão dos trabalhos selecionados. Assim como consta na Figura 1.

Figura 1 - Etapas para desenvolvimento da pesquisa.

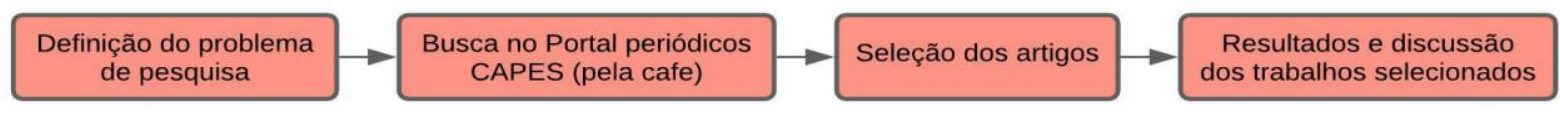

Fonte: Autores.

Detalhamento de cada etapa do desenvolvimento da pesquisa:

\section{Definição do problema da pesquisa}

A presente pesquisa pretende responder aos seguintes questionamentos: "o tijolo de solo-cimento apresenta uma viabilidade econômica e ambiental comparado ao tijolo cerâmico? a incorporação de resíduos ou materiais alternativos têm apresentado resultados satisfatórios?”, assim, buscar encontrar possíveis respostas, para as seguintes análises.

1. Análise do panorama da construção civil no Brasil, com uma análise dos fatores que avaliam esse setor da economia brasileira, assim como uma abordagem sobre os resíduos e materiais alternativos, suas definições e exposição do quadro de geração de resíduos dos municípios brasileiros. Por fim, um balanço sobre o déficit habitacional no Brasil, seu panorama e programas existentes com objetivo de tentar diminuir o quadro atual.

2. Analisar o tijolo de solo-cimento, suas definições, características e padrões normativos; comparar a viabilidade econômica e financeira com o tijolo cerâmico.

3. Analise dos estudos com tijolo de solo-cimento e a incorporação de resíduos ou materiais alternativos diversos e seus resultados.

\section{Busca no Portal periódicos CAPES, por meio da comunidade acadêmica federada (cafe)}

Foi utilizado o Portal periódicos CAPES, por meio da comunidade acadêmica federada (cafe) para a busca dos artigos da presente pesquisa.

\section{Seleção dos artigos}

Para a seleção dos artigos foi obedecido as seguintes etapas: utilização do descritor e aplicação dos critérios de inclusão e exclusão. O descritor utilizado foi “tijolo de solo-cimento". Em relação aos critérios de inclusão, foram selecionados artigos publicados nos últimos 6 anos, os quais correspondem aos anos de 2016 a 2022. Já para o critério de exclusão, foram excluídos artigos que não são de língua inglesa ou portuguesa, foram excluídos artigos de acordo com a leitura e seleção dos títulos, após foram excluídos artigos após a leitura e seleção dos resumos e após a leitura e seleção dos artigos na íntegra.

Portanto, seguindo as etapas mencionadas, foram encontrados 97 resultados de acordo com a busca pelo descritor, sendo 
selecionado e aceito apenas 20, a esquematização da seleção dos artigos é apresentada na Figura 2. Com a utilização do descritor “tijolo de solo-cimento" foram encontrados 97 resultados. Com o critério de inclusão dos artigos de 2016 a 2022, a busca obteve 49 resultados. Utilizando os critérios de exclusão, 7 trabalhos foram excluídos por não estarem na língua inglesa ou portuguesa, ficando com 42 resultados; 12 trabalhos foram excluídos após a leitura do título, ficando com 30 resultados; 3 trabalhos foram excluídos após a leitura dos resumos, ficando com 27 resultados; 7 trabalhos foram excluídos após a leitura dos artigos na íntegra, ficando com 20 resultados.

Figura 2 - Esquematização da metodologia de seleção dos artigos.

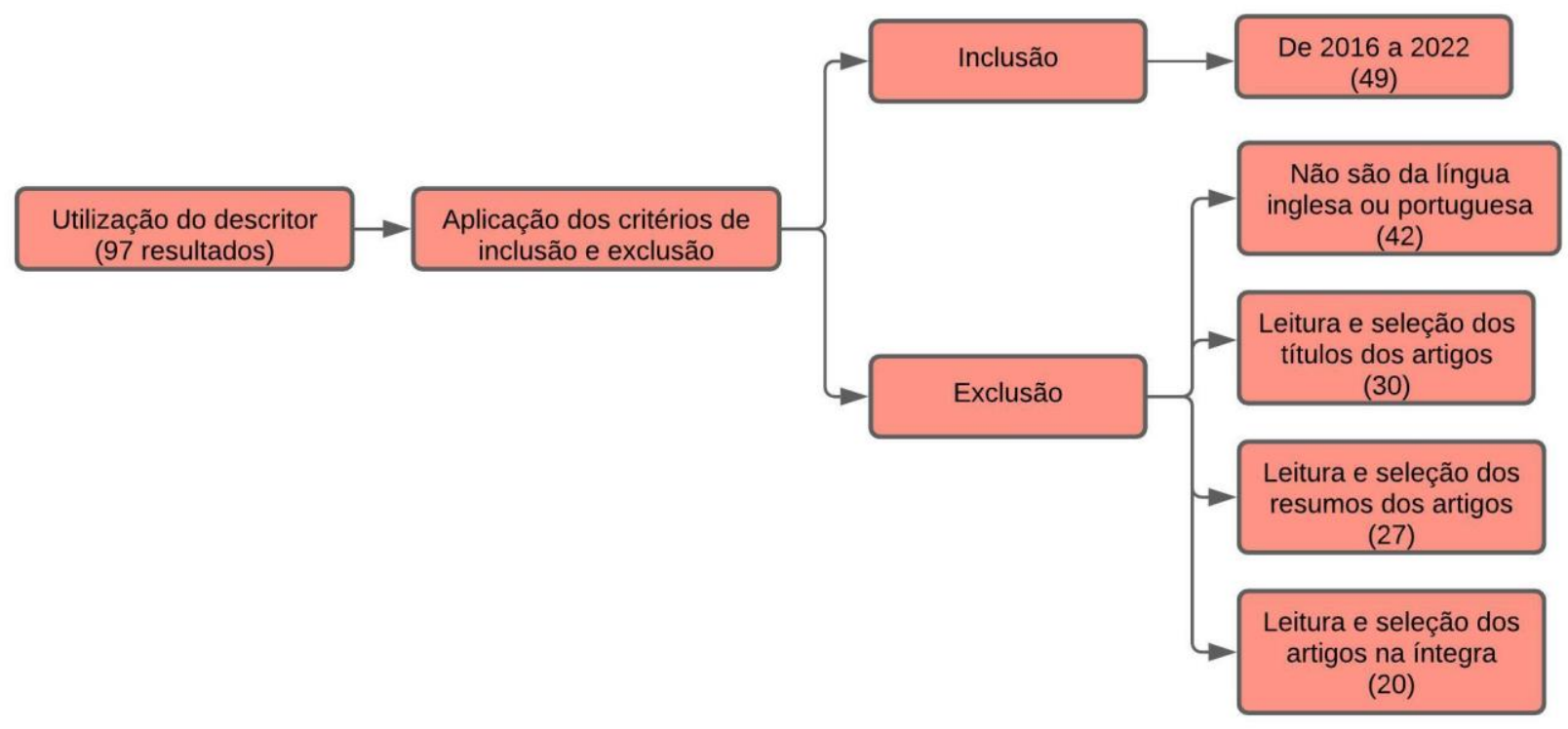

Fonte: Autores.

\section{Resultados e discussão dos trabalhos selecionados}

Após a seleção dos artigos, foi feita uma discussão com os autores acerca da análise do panorama da construção civil no Brasil; análise do tijolo de solo-cimento, suas características e comparação com o tijolo cerâmico; análise da incorporação de resíduos ou materiais alternativos diversos, no tijolo de solo-cimento, e seus resultados.

\section{Resultados e Discussão}

Neste capítulo é apresentado os resultados e discussão da pesquisa, que versem sobre os seguintes temas: construção civil no Brasil; o tijolo de solo-cimento e um comparativo com o tijolo cerâmico; análise de pesquisas com tijolo de solo-cimento e a incorporação de resíduos ou materiais alternativos diversos e seus resultados.

\subsection{A construção civil no Brasil}

A Câmara Brasileira de Indústria da Construção (CBIC) descreve que a economia brasileira é dividida em três amplos setores, sendo esses da Agropecuária, Indústria e Serviços, com a Construção Civil pertencente ao setor da Indústria (CBIC, 2020).

O principal indicador para medir a representatividade do setor da Indústria e da Construção Civil na economia nacional, é a porcentagem do Produto Interno Bruto (PIB) de um país que esse segmento representa. O Instituto Brasileiro de Geografia e 
Estatística - IBGE (2020) define o PIB de um país como a soma de todos os bens e serviços finais que são produzidos por este país durante certo período, que geralmente é de um ano.

Os dados da CBIC (2020) referentes às Contas Nacionais Trimestrais $1^{\circ}$ Trim./2021, extraídos do IBGE, mostram que o PIB do Brasil em 2020 foi de 7,45 trilhões, com o setor da indústria responsável por 20,4\%, e a construção civil por 3,3\%.

Os dados do parágrafo anterior demonstram como o setor da construção civil é responsável pela economia de um país e o quanto ela influencia na geração e descarte de resíduos em larga escala, consumindo e degradando o meio ambiente. Sobre esse aspecto, Pisani $(2005$, p. 53) salienta que "não existe construção que não gere impacto, a busca é por intervenções que o ocasionam em menor escala".

O Conselho Nacional de Meio Ambiente (CONAMA), por meio da Resolução n 307, estabelece diretrizes, critérios e procedimentos para a gestão dos resíduos da construção civil (RCC), definindo-os como "os provenientes de construções, reformas, reparos e demolições de obras de construção civil, e os resultantes da preparação e da escavação de terrenos". Os RCC são comumente também chamados de entulhos de obras (Brasil, 2002).

Ainda de acordo com a Resolução n 307, o CONAMA classifica os RCC em classes, como as descritas a seguir: Classe A: resíduos reutilizáveis ou recicláveis como agregados; Classe B: resíduos recicláveis para outros destinos; Classe C: resíduos em que não foram desenvolvidas tecnologias ou aplicações viáveis economicamente permitindo sua reciclagem ou recuperação; Classe D: resíduos perigosos provenientes do processo de construção (Brasil, 2002). Na Figura 3 é possível observar resíduos da construção civil (Classe A), em (a) resíduo proveniente de rochas e (b) resíduo cerâmico.

Figura 3 - Resíduos da construção civil, em (a) resíduo proveniente de rocha e em (b) resíduo cerâmico.
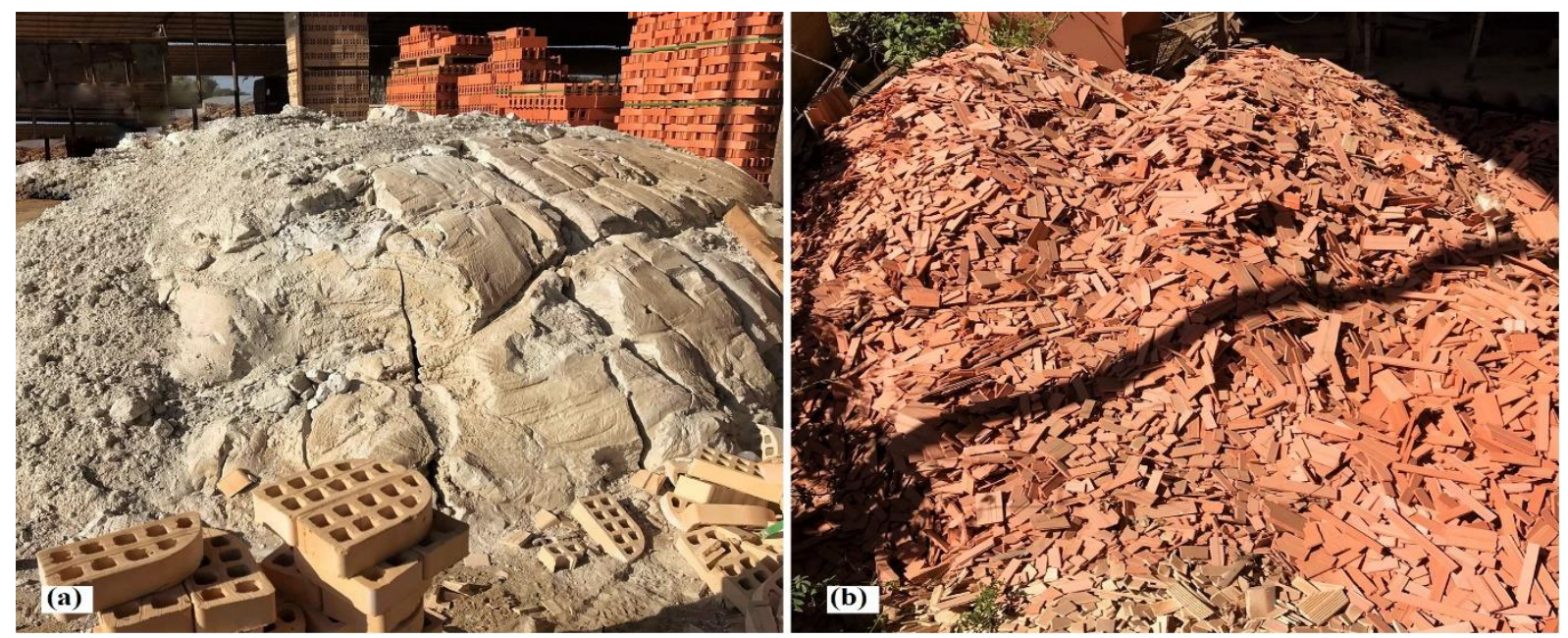

Fonte: Autores.

De acordo com a Associação Brasileira de Limpeza Pública e Resíduos Especiais (ABRELPE), em 2019, os municípios brasileiros coletaram um total de 44,5 milhões de toneladas de Resíduos de Construção e Demolição (RCD), o que resulta em uma coleta per capita de 213,5 kg/hab/ano; em relação às regiões do Brasil, do total coletado, 52,19\% correspondem à região Sudeste, $19,77 \%$ à região Nordeste, 13,32\% à região Sul, 10,86\% à região Centro-Oeste e 3,86\% à região Norte (ABRELPE, 2020). Ao levar em consideração que os dados da pesquisa mencionada não incluem os resíduos coletados por empresas privadas, uma vez que os dados foram frutos da pesquisa direta da ABRELPE e dos municípios, percebe-se que há um quantitativo muito maior na geração dos RCD.

Na construção civil, além dos problemas ambientais, há o problema do déficit habitacional no Brasil; o estudo intitulado "Déficit Habitacional no Brasil", publicado pela Fundação João Pinheiro (FJP), relata que o déficit habitacional estimado no 
território Brasileiro para o ano de 2019 foi de 5,876 milhões de domicílios; o que representa, em percentual relativo, 8\% do total de domicílios particulares permanentes e improvisados do país (FJP, 2021).

A Figura 4 retrata o percentual relativo do déficit habitacional decorrente das unidades da Federação - Brasil dos anos de 2016 a 2019, do qual é possível concluir que as regiões Nordeste e Norte são as regiões que possuem o maior déficit em termos relativos e os estados do Maranhão, Roraima e Amapá são um dos estados que possuem o maior déficit relativo (FJP, 2021).

Figura 4 - Percentual relativo do déficit habitacional decorrente das unidades da Federação - Brasil dos anos de 2016 a 2019.
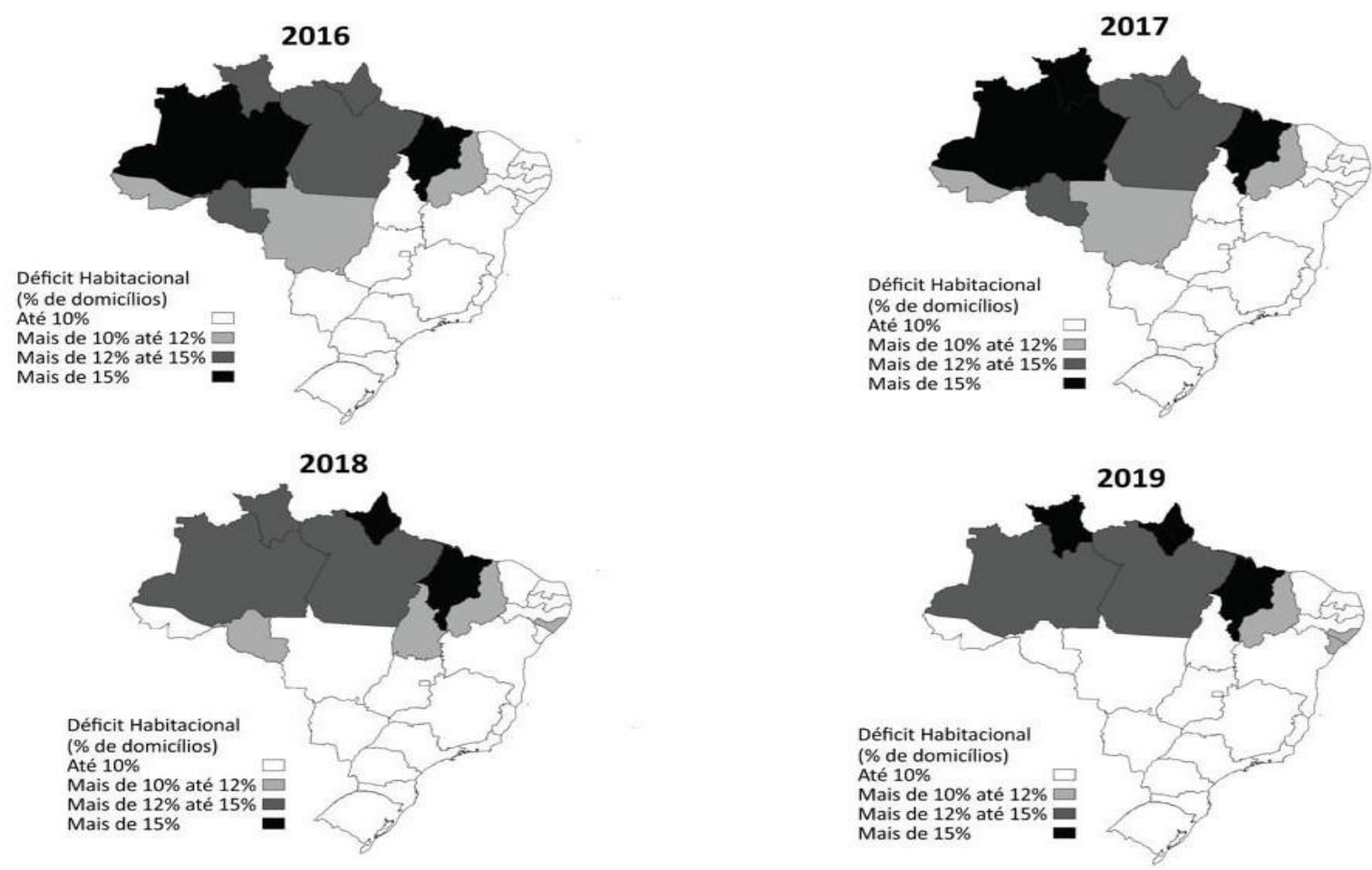

Fonte: FJP (2021).

Existem programas destinados à habitação social no país, como Minha Casa Minha Vida (MCMV), Pró-Moradia, Agência Paulista de Habitação Social (Casa Paulista) e Companhia de Desenvolvimento Habitacional e Urbano de São Paulo (CDHU), além de parcerias privadas e públicas; no entanto, ao olhar para os levantamentos estatísticos da FJP (2021) e suas perspectivas, percebe-se que ainda não são suficientes, uma vez que houve um aumento no déficit habitacional de 2016 para 2019 (4\%) e é esperado um aumento para 2021 e próximos anos.

Em meio a esse cenário de grande preocupação ambiental, dos impactos gerados pela construção civil ao meio ambiente, quer seja pelo consumo de recursos naturais ou pela geração de resíduos da construção civil, aliado ao problema do déficit habitacional no Brasil, a estabilização de solo com destino à utilização na construção civil, como na produção de tijolos de solocimento, surge como uma alternativa viável, factível e de menor impacto ambiental face aos métodos construtivos já consolidados.

\subsection{O tijolo de solo-cimento}

O tijolo de solo-cimento é definido pela NBR 8491 (Associação Brasileira De Normas Técnicas, 2012) como sendo um componente de alvenaria que é formado por uma mistura homogênea, compactada e endurecida de solo, cimento Portland e água e, ocasionalmente, pigmentos e/ou aditivos com proporções que atendem aos requisitos da referida norma. $\mathrm{O}$ tijolo possui a 
altura em menor dimensão que a largura e pode ser maciço, quando seu volume é igual ou superior a 85\% do volume aparente, ou vazado, quando for inferior a 85\%, apresentando furos verticais (Associação Brasileira De Normas Técnicas, 2012). Observase na Figura 5, exemplo de tijolo vazado (1) e maciço (2) de solo-cimento.

Figura 5 - Tijolo vazado e maciço de solo-cimento.

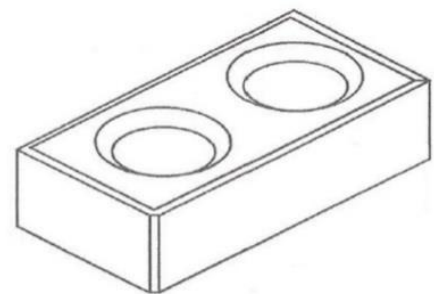

(1)

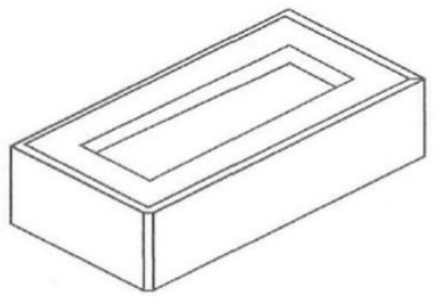

(2)

Fonte: Adaptada de NBR 8491 (Associação Brasileira De Normas Técnicas, 2012).

A NBR 10833 (Associação Brasileira De Normas Técnicas, 2013) determina os requisitos gerais, em função dos constituintes do solo-cimento; o solo precisa apresentar $100 \%$ do material passante na peneira $4,75 \mathrm{~mm}\left(\mathrm{n}^{\circ} 4\right)$ e 10 a $50 \%$ na peneira $75 \mu \mathrm{m}\left(\mathrm{n}^{\circ} 200\right)$, ter o limite de liquidez e o índice de plasticidade menores ou iguais, respectivamente, a $45 \%$ e $18 \%$ e não conter material orgânico em quantitativo que vá prejudicar o processo de hidratação do cimento; o cimento precisa atender às exigências normativas de acordo com o seu tipo; a água deve ser isenta de impurezas que são nocivas à hidratação do cimento; é permitido o uso de aditivos e pigmentos, desde que o tijolo atenda aos requisitos físicos mecânicos prescritos em normas.

A NBR 10833 (Associação Brasileira De Normas Técnicas, 2013) determina sobre o processo de fabricação do tijolo de solo-cimento, conforme é demonstrado pela Figura 6, ainda de acordo com a referida norma, a etapa de mistura do solocimento, podendo ser feita de forma manual ou mecânica, se dá ao adicionar cimento ao solo, já destorroado e peneirado, misturando até atingir uma cor uniforme. Em seguida é colocado água de forma gradual até atingir a umidade ideal de trabalho. Na etapa de moldagem é recomendado transferir, rapidamente, a mistura para o molde e realizar a prensagem, retirando os tijolos e empilhando-os em um local com sombra em uma superfície lisa e plana, obedecendo a altura máxima de um metro e meio. Durante os sete primeiros dias, deve-se manter os elementos úmidos, para a realização da cura. A utilização dos tijolos só é permitida após 14 dias de sua fabricação. Após a cura, é recomendado o uso de paletes para o armazenamento e transporte dos tijolos.

Figura 6 - Processo de fabricação do tijolo de solo-cimento.

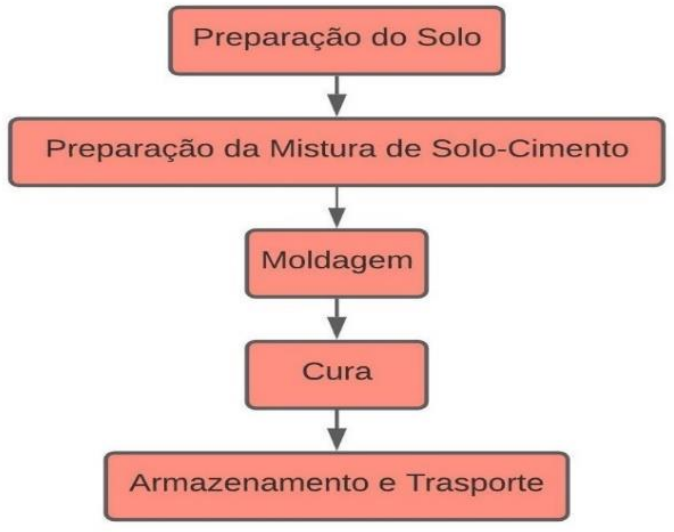

Fonte: Adaptada de NBR 10833 (Associação Brasileira De Normas Técnicas, 2013). 
Para avaliar as propriedades tecnológicas dos tijolos de solo-cimento, a NBR 8492 (Associação Brasileira De Normas Técnicas, 2012) descreve os métodos de ensaios, sendo eles de análise dimensional, resistência à compressão simples e absorção de água. Sobre esse assunto, a NBR 8491 (Associação Brasileira De Normas Técnicas, 2012) apresenta os requisitos normativos para os ensaios, sendo permitido tolerâncias de +1 ou -1 mm nas dimensões nominais para as três dimensões dos tijolos; com idade mínima de 7 dias de cura, a média dos valores da resistência à compressão não podem ser menores que $2 \mathrm{MPa}$, nem valor individual menor que 1,7 MPa; para a absorção de água, com idade mínima de 7 dias de cura, a amostra ensaiada não pode apresentar a média dos valores maior do que $20 \%$, nem valores individuais maiores do que $22 \%$.

\subsubsection{Comparativo do tijolo de solo-cimento com o tijolo cerâmico}

Os estudos publicados por Kozloski et al. (2019), em que se estimou a quantificação de emissão de $\mathrm{CO}_{2}$ de materiais empregados na construção de duas residências de igual projeto arquitetônico, considerando a emissão de cada material, o quantitativo e o fator de perda. A utilização de tijolos de solo-cimento apresentou uma redução de 29,5\% nas emissões de $\mathrm{CO}_{2}$ comparado com os tijolos cerâmicos. Os autores acreditam que essa redução se dá, especialmente, por conta do processo de fabricação de ambos, uma vez que o tijolo de solo-cimento é prensado, não utiliza o processo de queima, assim como o tijolo cerâmico.

Em seu trabalho, Negreiros et al. (2018), traz considerações a respeito da comparação dos custos de uma construção de 40,16 m² voltada para Habitação de Interesse Social (HIS), ao utilizarem o tijolo de solo-cimento no projeto da construção da alvenaria e acabamento, os autores tiveram uma economia próxima de $41 \%$, ao comparar com o tijolo cerâmico, levando em conta a mão de obra e os materiais.

Ainda de acordo com os resultados de Negreiros et al. (2018) percebe-se que o preço unitário do tijolo de solo-cimento é mais caro e que para o mesmo projeto houve a necessidade da utilização de mais tijolos, ficando no total 6,7 vezes mais caro. Para o revestimento da alvenaria em tijolo de solo-cimento os autores citaram a utilização de cola branca PVA, rejunte cerâmico flexível e resina acrílica impermeabilizante, já para o tijolo cerâmico comum, foi cotado a utilização do chapisco, reboco, argamassa para revestimento, massa acrílica e tinta e seladores, obtendo assim um custo de 12,35 vezes mais caro a utilização do tijolo cerâmico. Em relação ao tempo de execução da mão de obra, para o tijolo de solo-cimento é projetado um tempo de 1,91 vezes mais rápido, e assim uma economia de mesma escala.

\subsection{Análise de pesquisas com tijolo de solo-cimento e a incorporação de resíduos ou materiais alternativos diversos e seus resultados}

Há muitas pesquisas sobre a viabilidade da incorporação de diferentes resíduos ou de produtos alternativos no solocimento, com o objetivo de ter um reaproveitamento de resíduo e consequentemente uma redução ao conceder uma destinação final para o resíduo. Esses resíduos substituem parcialmente o solo da mistura ou o aglomerante, que no caso do solo-cimento é o cimento. Quando os resíduos ou materiais alternativos substituem o cimento, além do reaproveitamento dos resíduos ou materiais alternativos, evitando acúmulo em certas áreas e o descarte irregular, existe também a vantagem de diminuir o quantitativo do cimento na mistura.

Felix e Possan (2018) apontam que o Brasil é um dos principais países que mais consome e produzem cimento na América Latina, atingindo a quantia de quase 70 milhões de toneladas nos últimos anos. Existe uma previsão de produção mundial de cimento, realizada pela IEA - International Energy Agency (2009), para o ano de 2050 de 3,69x109 de toneladas por ano e $5 \times 10^{9}$ em uma situação de crescimento elevado (Müller \& Harnisch, 2008).

Na visão de Mehta e Monteiro (2014), o consumo do concreto, em esfera mundial, tem uma tendência de crescimento por ano, em consequência do cimento também. A mesma tendência teórica exposta anteriormente é compartilhada por (IEA, 
2009), ao afirmar que mesmo que se teve algum sucesso na redução de $\mathrm{CO}_{2}$ devido a substituição do clínquer e ganho energético dos fornos, as emissões de $\mathrm{CO}_{2}$, advindas da produção de cimento têm crescido a contar de 1940. Damineli e John (2012) destacam que o crescimento da produção de concreto é esperado com intenção de auxiliar o crescimento dos países em desenvolvimento. Para aumentar o problema, os autores acrescentam que não existem adições de minerais suficientes de forma que possa aumentar os índices de substituição de clínquer.

Logo, é necessário medidas e posturas alternativas para diminuir a utilização do cimento, como por exemplo verificando a viabilidade da substituição do cimento por outros materiais e também opções para com o destino final de resíduos e materiais alternativos, como o aproveitamento em tijolos de solo-cimento. Nesse sentido, é possível encontrar na literatura a incorporação de borracha, garrafa pet, lodo da indústria de papel e celulose, materiais recicláveis da coleta seletiva urbana, resíduos da construção civil, de atividade industriais e de coprodutos siderúrgicos, além de produtos alternativos como casca de café, de banana, de arroz e fibra vegetal (Ribeiro et al., 2021); (Silva et al., 2021); (Tosello et al, 2021); (Lima \& Lima, 2020); (Azevedo et al. 2019); (Castro et al., 2019); (Cristina et al., 2018); (Silva \& Aguiar, 2017).

No Erro! Fonte de referência não encontrada. abaixo é possível obter um resumo sobre pesquisas de utilização de resíduos ou materiais alternativos no solo-cimento, seus autores, as características avaliadas e os resultados obtidos.

Quadro 1 - Pesquisas com a incorporação de diversos resíduos no solo-cimento.

\begin{tabular}{|c|c|c|c|}
\hline Autores & Resíduo utilizado & Características avaliadas & Principais resultados \\
\hline Ribeiro et al. (2021) & Borracha de pneu & Resistência à compressão e absorção & $\begin{array}{c}\text { Não aprovados a resistência à compressão e } \\
\text { absorção de água }\end{array}$ \\
\hline Silva et al. (2021) & Garrafa pet triturada & Resistência à compressão e absorção & $\begin{array}{l}\text { Os traços apresentaram resultados favoráveis } \\
\text { para absorção de água, mas não para } \\
\text { resistência à compressão. }\end{array}$ \\
\hline Tosello et al. (2021) & $\begin{array}{c}\text { Materiais recicláveis da coleta } \\
\text { seletiva urbana (PET e EPS), } \\
\text { reboco descartado e vinhaça } \\
\text { da indústria canavieira }\end{array}$ & $\begin{array}{l}\text { Absorção de água e compressão } \\
\text { simples }\end{array}$ & $\begin{array}{l}\text { Com a inclusão dos excessivos materiais não } \\
\text { foi possível atingir os padrões mínimos } \\
\text { normativos }\end{array}$ \\
\hline Lima e Lima (2020) & $\begin{array}{c}\text { Resíduo de argamassa de } \\
\text { assentamento e revestimento }\end{array}$ & $\begin{array}{l}\text { Resistência à compressão, absorção e } \\
\text { durabilidade }\end{array}$ & $\begin{array}{l}\text { Apresentou resultados favoráveis com } 20 \% \\
\text { de resíduo em substituição ao solo }\end{array}$ \\
\hline Vilela et al. (2020) & $\begin{array}{l}\text { Resíduos de mineração de } \\
\text { minério de ferro }\end{array}$ & $\begin{array}{l}\text { Densidade seca, perda de massa por } \\
\text { imersão, resistência à compressão, } \\
\text { condutividade térmica e } \\
\text { microestrutura. }\end{array}$ & $\begin{array}{l}\text { Influência positiva na densidade; resistência } \\
\text { à compressão, sendo com } 10 \% \text { de resíduo foi } \\
\text { o melhor; condutividade térmica com relação } \\
\text { com a densidade, maior condutividade } \\
\text { quanto maior a densidade }\end{array}$ \\
\hline Azevedo et al. (2019) & $\begin{array}{l}\text { Lodo da indústria de papel e } \\
\text { celulose }\end{array}$ & $\begin{array}{l}\text { Resistência à compressão, absorção de } \\
\text { água e durabilidade }\end{array}$ & $\begin{array}{l}\text { A incorporação de resíduos de até } 10 \% \\
\text { atendeu aos requisitos técnicos normativos }\end{array}$ \\
\hline Castro et al. (2019) & $\begin{array}{c}\text { Casca de café em substituição } \\
\text { parcial ao cimento }\end{array}$ & $\begin{array}{l}\text { Resistência à compressão, absorção de } \\
\text { água e isolante térmico }\end{array}$ & $\begin{array}{l}\text { De modo geral os materiais não se } \\
\text { enquadraram nos padrões normativos }\end{array}$ \\
\hline Cristina et al. (2018) & Fibra vegetal & Resistência à compressão e absorção & $\begin{array}{l}\text { Aprovados em absorção de água e } \\
\text { reprovados em resistência à compressão }\end{array}$ \\
\hline Autores & Resíduo utilizado & Características medidas & Principais resultados \\
\hline Ferreira e Cunha (2017) & $\begin{array}{c}\text { Resíduos das cascas de arroz e } \\
\text { de braquiária }\end{array}$ & $\begin{array}{l}\text { Absorção de água e compressão } \\
\text { simples }\end{array}$ & $\begin{array}{l}\text { Ensaio de absorção atendeu os padrões } \\
\text { normativos, e o resistência à compressão no } \\
\text { sem resíduo, } 10 \% \text { e } 20 \% \text {. }\end{array}$ \\
\hline Leonel et al (2017) & $\begin{array}{l}\text { Areia descartada de fundição } \\
\text { (ADF) }\end{array}$ & $\begin{array}{c}\text { Absorção de água e compressão } \\
\text { simples }\end{array}$ & $\begin{array}{c}\text { A areia descartada de fundição reduziu a } \\
\text { absorção e manteve a resistência à } \\
\text { compressão em níveis aceitáveis para tijolo } \\
\text { de solo-cimento }\end{array}$ \\
\hline Silva e Aguiar (2017) & $\begin{array}{l}\text { Casca de Banana em } \\
\text { substituição parcial ao } \\
\text { cimento }\end{array}$ & $\begin{array}{l}\text { Absorção de água e compressão } \\
\text { simples }\end{array}$ & Não atendeu os padrões normativos \\
\hline Castro et al. (2016) & $\begin{array}{l}\text { Coprodutos siderúrgicos: pó } \\
\text { de balão, poeiras de } \\
\text { despoeiramento de aciaria } \\
\text { elétrica e escória de forno } \\
\text { elétrico a arco }\end{array}$ & $\begin{array}{c}\text { Resistência à compressão e absorção } \\
\text { de água }\end{array}$ & $\begin{array}{c}\text { Resultados mostram a potencialidade do uso } \\
\text { do uso de resíduos siderúrgicos, verificando } \\
\text { a possibilidade de bons resultados }\end{array}$ \\
\hline Siqueira et al (2016) & $\begin{array}{l}\text { Resíduos de atividades } \\
\text { industriais: casca de ovo } \\
\text { galináceo e escória de } \\
\text { soldagem } \\
\end{array}$ & $\begin{array}{l}\text { Retração volumétrica, absorção de } \\
\text { água, densidade aparente, } \\
\text { durabilidade e resistência à } \\
\text { compressão simples }\end{array}$ & $\begin{array}{l}\text { Até } 15 \% \text { de resíduo de escória de fluxo de } \\
\text { soldagem e até } 30 \% \text { em massa de resíduo de } \\
\text { casca de ovo galináceo podem ser utilizados } \\
\text { para a fabricação de tijolos de solo-cimento }\end{array}$ \\
\hline
\end{tabular}

Fonte: Autores. 


\section{Considerações Finais}

A busca por materiais de menor impacto ambiental, que apresentam uma diminuição da emissão de poluentes na atmosfera, que possibilitam uma redução ou reaproveitamento de resíduos, com uma boa relação custo-benefício e que possam ser utilizados na construção civil de forma a cumprir os requisitos técnicos que as normas estabelecem, em função da finalidade de cada composição, são pautas fundamentais para uma constante evolução da construção civil e sociedade, tendo se tornado cada vez mais recorrente.

O tijolo de solo-cimento, por apresentar uma vantagem financeira na execução dos projetos - economia de $41 \%$ comparado ao tijolo cerâmico (Negreiros et al., 2018), pode ser articulado com as habitações de interesse social, sendo usado para construir mais habitações e melhorar as projeções do déficit habitacional no Brasil; além disso, gera menos impacto ambiental, seja pela diminuição dos gases poluentes - redução de 29,5\% de $\mathrm{CO}_{2}$ comparado ao tijolo cerâmico (Kozloski et al., 2019), ou pelo reaproveitamento de resíduos.

Sobre o reaproveitamento de resíduos, é possível encontrar na literatura a incorporação de borracha, garrafa pet, lodo da indústria de papel e celulose, materiais recicláveis da coleta seletiva urbana, resíduos da construção civil, de atividade industriais e de coprodutos siderúrgicos, além de produtos alternativos como casca de café, de banana e fibra vegetal (Ribeiro et al., 2021); (Silva et al., 2021); (Tosello et al, 2021); (Lima \& Lima, 2020); (Azevedo et al. 2019); (Castro et al., 2019); (Cristina et al., 2018); (Silva \& Aguiar, 2017).

Os resultados da adição de borracha de pneu, no caso de Ribeiro et al. (2021), não atingiram os padrões normativos de todos os ensaios predeterminados pelas normas de solo-cimento; assim como na incorporação de garrafa pet triturada proposta por Silva et al. (2021); na pesquisa de Tosello et al. (2021), ao adicionar materiais recicláveis da coleta seletiva urbana como PET e EPS, além de reboco descartado e vinhaça da indústria canavieira também não foi possível obter os padrões normativos; de igual forma nos estudos de Silva e Aguiar (2017) e Cristina et al. (2018) ao incorporar casca de banana em substituição ao cimento e adicionar fibra vegetal em uma mistura de solo-cimento, respectivamente.

Já os resultados de Lima e Lima (2020) foram positivos com a incorporação de resíduo de argamassa de assentamento e revestimento para o traço com $20 \%$ de resíduo em substituição ao solo, nos estudos de Castro et al. (2016) foi possível verificar a possibilidade de bons resultados para o uso de resíduos siderúrgicos no tijolo de solo-cimento, mostrando o seu potencial de uso.

Apesar de alguns resultados ainda não estão de acordo com os padrões normativos, quando se trata de solo-cimento, a forma como cada componente é adicionado na mistura, seja por substituição parcial ao solo ou ao cimento, ou apenas adição, aliado a outros fatores que influenciam no resultado, como o solo utilizado, o tipo de cimento, fatores como a umidade da mistura no momento da prensagem e a força de prensagem utilizada, a utilização de prensa manual ou hidráulica, umidade produzida e a metodologia de mistura, torna esse tema inesgotável de possibilidades, demonstrando a diversidade e potencialidade do tijolo de solo-cimento. Potencial esse que fica evidente por diversos fatores, como o reaproveitamento de resíduos ou materiais alternativos, podendo auxiliar no descarte e reaproveitamento desses materiais; por ter um menor custo financeiro total da obra e menor emissão de $\mathrm{CO}_{2}$ comparado ao tijolo cerâmico, tornando a construção um pouco menos agressiva ao meio ambiente; além de ter potencial para ser utilizado em programas habitacionais com intuito de diminuir o déficit habitacional no Brasil.

Sugere-se, para trabalhos futuros, o desenvolvimento de pesquisas experimentais, utilizando resíduos que ocasionam problemas em relação aos seus descartes, de forma a incorporá-los em tijolos de solo-cimento. O objetivo de tais pesquisas seria dar um destino adequado para esses resíduos e, assim, desenvolver um tijolo que atenda aos padrões normativos de tijolo de solo-cimento, podendo ser utilizado na construção civil. 


\section{Agradecimentos}

O presente trabalho foi realizado com apoio da Coordenação de Aperfeiçoamento de Pessoal de Nível Superior - Brasil (CAPES) - Código de Financiamento 001.

\section{Referências}

ABRELPE. (2020). Panorama dos resíduos sólidos no Brasil. Associação Brasileira de Limpeza Pública e Resíduos Especiais.

Associação Brasileira de Normas Técnicas. NBR 8491 (2012). Tijolo de solo-cimento - Requisitos.

Associação Brasileira de Normas Técnicas. NBR 8492 (2012). Tijolo de solo-cimento - Análise dimensional, determinação da resistência à compressão e da absorção de água - Método de ensaio.

Associação Brasileira de Normas Técnicas. NBR 10833 (2013). Fabricação de tijolo e bloco de solo-cimento com utilização de prensa manual ou hidráulica Procedimento.

Azevedo, A. R. G., Marvila, T. M., Fernandes, W. J., Alexandre, J., Xavier, G. C., Zanelato, E. B., \& Mendes, B. C. (2019). Assessing the potential of sludge generated by the pulp and paper industry in assembling locking blocks. Journal of Building Engineering, 23, 334-340.

Brasil. Conselho Nacional do Meio Ambiente (CONAMA). Resolução no 307. Diário Oficial da União. Imprensa Oficial, 5, 2002.

Brasil. (2020). Produto Interno Bruto - PIB. IBGE. https://www.ibge.gov.br/explica/pib.php.

Castro, E., Villella, L., Mendes, L., Mendes, R., Ribeiro, A., Júnior, J. \& Rabelo, G. (2019). Analysis of the coffee peel application over the soil-cement bricks properties. Coffee Science, 14(1), 12.

Castro, M., Costa, F., Borba, S., Fagury Neto, E., \& Rabelo, A. (2016). Avaliação das propriedades físicas e mecânicas de blocos de solo-cimento formulados com coprodutos siderúrgicos. Matéria, 21(3), 666-676.

CBIC. (2020). PIB Brasil e Construção Civil. http://www.cbicdados.com.br/menu/pib-e-investimento/pib-brasil-e-construcao-civil.

International Energy Agency. (2009). Cement Technology Roadmap: carbon emissions reductions up to 2050. Organisation for Economic and Co-operation and Development.

Cristina, P., Salomão, P., Cangussú, L., \& Carvalho, P. (2018). Tijolo solo cimento com adição de fibra vegetal: Uma alternativa na construção civil. Research, Society and Development, 7(9), 779439.

Damineli, B. L., \& John, V. M. (2012). Developing low CO2 concretes: is clinker replacement sufficient? The need of cement use efficiency improvement. In Key Engineering Materials 517, 342-351. Trans Tech Publications Ltd.

Felix, E., \& Possan, E. (2018). Balance emissions and CO2 uptake in concrete structures: Simulation based on the cement content and type. Revista ibracon de estruturas e materiais, 11(1), 135-162.

Ferreira, R., \& Cunha, A. (2017). Quality evaluation of soil-cement-plant residue bricks by the combination of destructive and non-destructive tests. Revista Brasileira De Engenharia Agrícola e Ambiental, 21(8), 543-549.

FJP. (2021). Déficit Habitacional no Brasil - 2016-2019, Belo Horizonte.

Kozloski, C., Vaghetti, M., \& da Silva, B. (2019). Emissões de $\mathrm{co}_{2}$ na casa popular eficiente e o emprego de materiais alternativos. Euro elecs: III encuentro latinoamericano y europeo de Edificaciones y comunidades sostenibles.

Leonel, R., Folgueras, M., Dalla Valentina, L., Prim, S., Prates, G., \& Caraschi, J. (2017). Characterization of soil-cement bricks with incorporation of used foundry sand. Cerâmica (São Paulo), 63(367), 329-335.

Lima, F., \& Lima, P. (2020). Resumo de tese: blocos de terra compactada de solo-cimento com resíduo de argamassa de assentamento e revestimento: caracterização para uso em edificações. MIX Sustentável, 6(3), 183-184.

Mehta, P., monteiro, P., \& Concreto Microestrutura, P. (2014). Materiais. (2a ed.), IBRACON, 751.

Müller, N., \& Harnisch, J. (2008). How to Turn Around the Trend of Cement Related Emissions in the Developing World. WWF-Lafarge Conservation Partnership: Gland, Switzerland.

Negreiros, R. L., Nunes, K. G., Bispo, C., \& de Morais, A. (2018). Comparativo sustentável e econômico entre a utilização do tijolo solo-cimento e o tijolo cerâmico de vedação em habitação de interesse social na cidade de teófilo otoni-mg. Revista Multidisciplinar do Nordeste Mineiro-Unipac ISSN, $2178,6925$.

Pisani, M. A. J. (2005). Um material de construção de baixo impacto ambiental: O tijolo de solo-cimento. Sinergia, 6(1), 53-59.

Ribeiro, V., Werdine, D., Barbosa, L., Pinheiro, M., Oliveira, A., Alves, A., \& Silva, L. (2021). Tijolo solo cimento com acréscimo de resíduos de borracha de pneu. Research, Society and Development, 10(12), E253101220504.

Silva, D., \& Aguiar, M. B. (2017). A utilização da casca da banana como substituição de parte do cimento na produção de tijolos ecológicos: a busca por alternativas sustentáveis. Percurso acadêmico, 7(13), 19-32. 
Research, Society and Development, v. 11, n. 2, e19011225605, 2022

(CC BY 4.0) | ISSN 2525-3409 | DOI: http://dx.doi.org/10.33448/rsd-v11i2.25605

Silva, P. F., Sousa, R. M. L., das Chagas Oliveira, F., Melo, S. T., Sousa, H. F., Lopes, P. D., \& Soares, R. A. L. (2021). Estudo da viabilidade de utilização de garrafa PET triturada na produção de tijolos ecológicos. Research, Society and Development, 10(14), e455101422273.

Siqueira, F., Amaral, M., Bou-Issa, R., \& Holanda, J. (2016). Influence of industrial solid waste addition on properties of soil-cement bricks. Cerâmica (São Paulo), 62(363), 237-241.

Tosello, M. E. C., Tamashiro, J. R., Silva, L. H. P., Antunes, P. A., \& Simões, R. D. (2021). Influência de materiais recicláveis e vinhaça da cana de açúcar na resistência mecânica de tijolos ecológicos. Research, Society and Development, 10(2), e56910212911.

Vilela, A. P., Eugênio, T. M. C., de Oliveira, F. F., Mendes, J. F., Ribeiro, A. G. C., Brandão, L. E. V. D. S., \& Mendes, R. F. (2020). Technological properties of soil-cement bricks produced with iron ore mining waste. Construction and Building Materials, 262, 120883.

Whittemore, R., \& Knafl, K. (2005). The integrative review: updated methodology. Journal of advanced nursing, 52(5), 546-553. 